\title{
Toward Avoiding Energy Holes in UnderWater Acoustic Sensor Networks
}

\author{
Chaima Zidi*, ${ }^{*}$, Fatma Bouabdallah ${ }^{\ddagger}$, Raouf Boutaba and Ahmed Mehaoua* \\ ${ }^{*}$ LIPADE, University of Paris Descartes, Sorbonne Paris Cité, Paris, France \\ $\left.{ }^{\ddagger}\right)$ Faculty of Computing and Information Technology, Information Technology department, King Abdulaziz \\ University ; P.O. Box 42808, Jeddah 21551 \\ ( ) School of Computer Science, University of Waterloo ; 200 University Ave. W., Waterloo, ON, Canada \\ E-Mail: czidi@kau.edu.sa, fothman1@kau.edu.sa, rboutaba@uwaterloo.ca, ahmed.mehaoua@parisdescartes.fr
}

\begin{abstract}
UnderWater Acoustic Sensor Networks (UW-ASNs) require protocols that make judicious use of the limited energy budget of the underwater sensor nodes. In this paper, we tackle the problem of energy holes in UW-ASNs. We show that we can balance the energy consumption through the network provided that sensors can use multiple transmission ranges when they send or forward the periodically generated data. In particular, we suppose that sensors can adjust their communication ranges up to three possible levels and we determine the set of possible next hops with the associated load weights that lead to a fair energy consumption among all underwater sensors. Hence energy holes can be avoided and consequently the network lifetime is highly increased.

Index Terms - UnderWater Acoustic Sensor Networks, sink
\end{abstract} hole problem, energy saving.

\section{INTRODUCTION}

Underwater Acoustic Sensor Networks (UW-ASNs) have gained an increasingly growing interest within the research community, due to their important applications. Indeed, UWASNs can be deployed to satisfy a broad underwater applications such as: offshore exploration, tsunami warning, and mine reconnaissance [1]. Fundamental differences between underwater acoustic propagation and terrestrial radio propagation imposes the design of new networking protocols.

The challenges in underwater communications are imposed by the severe underwater characteristics such as the highattenuation, bandwidth-limited underwater acoustic channel and limited battery power. Indeed, the energy budget of underwater sensors is not only restricted but even worse cannot be recharged. Indeed, notice that solar energy in underwater environment cannot be used to charge the battery power supply of sensors. Moreover, it is worth noting that, the needed power for acoustic underwater communications is much greater than in terrestrial radio ones. The reasons behind this can be summarized as follows. First, terrestrial radio communication relies on different physical layer technology (RF waves vs. acoustic waves). Second, underwater communication is subject to transmission over higher distances. Finally, to achieve underwater communication, more complex signal processing techniques are needed at the receivers to counterbalance the impairments of the channel.

Due to the aforementioned reasons, UW-ASNs necessitate communication protocols that judiciously use the limited energy budget of the underwater sensor nodes. For this purpose, load balancing is considered as a critical technique to extend the UW-ASN lifetime such that all the sensors drain their energy capacity as slowly and uniformly as possible. In terrestrial wireless sensor networks, it was proven that the nearest sensors to the sink tend to drain their supplied energy capacity much faster than other sensors [2]- [6]. This energy expenditure disequilibrium may severely decrease the network lifetime; and hence it should be avoided to the most possible extent.

In this work, a balanced routing scheme aiming at avoiding energy holes in UW-ASNs is proposed and evaluated. We aim at balancing the energy consumption among all underwater sensors. To do so, we consider an UW-ASN where the sensors are manually deployed according to a well designed deployment pattern. Our balanced routing solution decides the load weight for each possible sensor's next hops along with the appropriate transmission range that lead to a fair energy consumption throughout the network and thus overcoming the sink-hole problem.

This paper is organized as follows. Section II presents the network and energy model. We formulate the problem and describe our proposed solution in section III. The results are presented in Section IV, where we conduct a performance comparison study between our proposal and the nominal transmission range data forwarding scheme. Finally, we conclude this paper with a summary of our contributions.

\section{NETWORK AND ENERGY MODEL}

We consider a 2-dimensional shallow underwater sensor network. The deployment strategy presumes that sensors are anchored to the ocean bottom and equipped with a floating buoy. Once inflated, the buoy will push the sensor towards the ocean surface. Note that, in such deployment the shallow bottom-anchored sensors have a precise information of their geographical position at deployment time. In our model, we assume a circular sensor field of radius $R$ where the sink is placed at the center. The studied sensor field is supposed to be virtually partitioned into disjoint concentric bands called coronas of constant width $r$. Intuitively, the width of each corona can not exceed the maximum transmission range of the sensor node: $d_{t x-\max }$. Let us, consider $K$ to be the total number of coronas around the sink. $K$ can be written as 


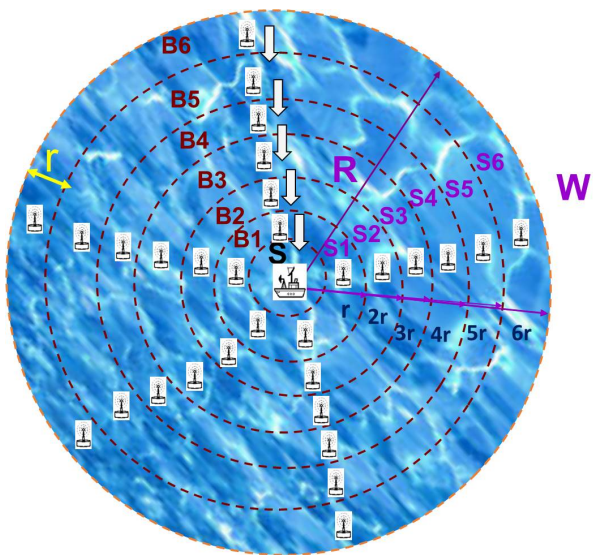

Fig. 1. Underwater Acoustic Sensor Network model.

follows:

$$
K=\left\lfloor\frac{R}{r}\right\rfloor
$$

For instance, in Fig. $1, K=6$, hence the sensor field can be seen as virtually partitioned into six coronas $B_{1}, B_{2}, B_{3}$, $B_{4}, B_{5}$ and $B_{6}$. Note that, the studied sensor network model is proposed in order to better approach the uniform energy depletion among all the sensors in the network.

In our work, we consider a continuous monitoring sensor application where the underwater sensors periodically generates $A$ reports per unit of time. Furthermore, we suppose that data reception and transmission are the main sources of energy consumption. Indeed, given that the deployment in underwater environment is quite sparse, the energy consumption due to overhearing can be neglected. More precisely, the energy spent in transmitting one packet of length $P_{l}$ bits over a distance $d$ is given by

$$
E_{t x}(d)=P_{T}(d) \times T_{t x}(d)
$$

where $P_{T}(d)$ is the transmission power over a distance $d$ and $T_{t x}(d)$ is the transmission time given by

$$
T_{t x}(d)=\frac{P_{l}}{C_{3 d B}(d)}
$$

where $C_{3 d B}(d)$ is the maximum allowed capacity over a bandwidth of $B_{3 d B}(d)$. Note that, in this paper, we use the bandwidth definition $B_{3 d B}(d)$ and the associated transmission power $P_{T}(d)$ and capacity definition $C_{3 d B}(d)$ as introduced in [7].

Likewise, the energy spent in receiving one $P_{l}$ bits packet is given by

$$
E_{r x}(d)=P_{r x}^{0} \times T_{t x}(d)
$$

where $P_{r x}^{0}$ is the electronics power.

According to the deployment pattern presented above, routing is quite intuitive. Indeed, each generated packet will cross adjacent coronas and hence forwarded by corresponding adjacent sensors till reaching the sink. For instance, Fig. 1 depicts a path along which a packet generated by a sensor located in the outermost corona is routed to the sink. Note that, along this example of path, each intermediate sensor node forward the packet to the immediately adjacent neighbor located the adjacent corona. Actually, our sensor field can be seen as a set of wedges. Each wedge $W$ is virtually partitioned into $K$ sectors, $S_{1}, S_{2}, \ldots, S_{K}$ by its intersection with $K$ concentric circles, centered at the sink, and of monotonically growing radius $r, 2 r, 3 r, \ldots, K r$, as shown in Fig. 1. Each sector contains exactly one sensor which has to forward the cumulative traffic coming from its predecessors in the downstream coronas to one of its possible successors in the upstream coronas. Specifically, in our study, we assume that each sensor is capable of adjusting its transmission range up to three levels. Each transmission range allows the sensor to reach one of the three upstream coronas. More precisely, the lowest transmission range allows each sensor to reach the immediately adjacent upstream sector, while the second highest transmission range allows it to reach the 2-hop away upstream corona and the highest transmission range allows the sensor to reach the 2-hop away upstream corona. Our work aims at deriving the appropriate load fractions for each possible upstream corona such that to balance the energy consumption among nodes in the same wedge $W$. More details are given in the next section.

\section{BALANCING ENERGY EXPENDITURE}

\section{A. Problem statement}

Recall that, in our study, all the sensor nodes transmit their periodically generated reports, $A$, to the sink node denoted by $S$. In this section we turn to the task of evaluating the energy consumption per sensor in an arbitrary corona $B_{i}$ with $i \geq 1$. Note that, according to our balanced routing scheme, every sensor in a given wedge $W$ and a generic corona $B_{i}$, $(1 \leq i \leq K)$, is asked to forward two kinds of reports:

- reports generated by an underwater sensor located in the same wedge $W$ but in a downstream corona $B_{j}$ with $i<j \leq$ $K$, and

- reports originating at the same sensor in $B_{i}$.

In this work, we strive for approaching the efficient routing of reports to the sink node by adequately distributing the traffic load on the individual sensor node such that a uniform and smooth energy consumption is guaranteed among all sensors in the network and hence the energy hole problem is overcome. For this purpose, we suppose that each underwater sensor is endowed with the ability to dynamically adjust its transmission range up to three possible levels.

In this study, as a first step, we suppose that for each sensor node located at corona $B_{i}$ in a specific wedge $W$, the next hop to send generated reports to the sink $S$ can be the sensor located in $B_{i-1}$ or $B_{i-2}$ in the same wedge $W$. In other words, we suppose that

$$
d_{t x-\max } \in\{r, 2 r\}
$$

Considering a wedge $W$, we associate to each possible next hop located in $B_{i-1}$ or $B_{i-2}$ a respective weight $\beta_{1}^{i}, \beta_{2}^{i}$ such that $\beta_{1}^{i}+\beta_{2}^{i}=1, \forall i, 1 \leq i \leq K$. Consequently, the total number of packets per unit of time, $A_{i}$, handled by the sensor in corona $B_{i}$ and wedge $W$, can simply be expressed as follows

$$
\begin{gathered}
A_{i}=A+\beta_{1}^{i+1} A_{i+1}+\beta_{2}^{i+2} A_{i+2} \\
\text { for } j=1,2 \text { if } i+j>K \text { then } \beta_{j}^{i+j}=0
\end{gathered}
$$


Consequently, the average transmission energy, $E_{t x}^{i}$, consumed by a sensor in corona $B_{i}$ and wedge $W$ can be derived as follows

$$
\begin{gathered}
E_{T X}^{i}=\beta_{1}^{i} A_{i} E_{t x}(r)+\beta_{2}^{i} A_{i} E_{t x}(2 r) \\
\text { for } j=1,2 \text { if } i-j<0 \text { then } \beta_{j}^{i}=0
\end{gathered}
$$

Likewise, the average reception energy, $E_{r x}^{i}$, consumed by a sensor in corona $B_{i}$ and wedge $W$ can be expressed as follows

$$
\begin{gathered}
E_{R X}^{i}=\beta_{1}^{i+1} A_{i+1} E_{r x}(r)+\beta_{2}^{i+2} A_{i+2} E_{r x}(2 r) \\
\quad \text { for } j=1,2 \text { if } i+j>K \text { then } \beta_{j}^{i+j}=0
\end{gathered}
$$

Finally, the total energy consumed by a sensor in corona $B_{i}$ and wedge $W$ is

$$
E^{i}=E_{T X}^{i}+E_{R X}^{i}
$$

The goal of our work is to tailor the coronas in such way that the energy expenditure is balanced across all the coronas. Consequently, our problem can be stated as follows:

$$
\begin{aligned}
& \text { given } K, r, d_{t x-\max } \\
& \text { Find } \beta_{1}^{i}, \beta_{2}^{i} \forall i, 1 \leq i \leq K \\
& \text { such that } E^{1}=E^{2}=\ldots=E^{K} \\
& \text { subject to } \\
& \beta_{1}^{i}+\beta_{2}^{i}=1, \forall i, 1 \leq i \leq k
\end{aligned}
$$

As a second step, we assume that each sensor node is not only able of sending up to $2 r$ away neighbor but it can also directly reach $3 r$ away neighbor. In other words, we suppose that,

$$
d_{t x-\max } \in\{r, 2 r, 3 r\}
$$

Consequently, in the same way we can derive the above expressed equations respectively as follows:

$$
\begin{gathered}
A_{i}=A+\beta_{1}^{i+1} A_{i+1}+\beta_{2}^{i+2} A_{i+2}+\beta_{3}^{i+3} A_{i+3} \\
\quad \text { for } j=1,2,3 \text { if } i+j>K \text { then } \beta_{j}^{i+j}=0 \\
E_{T X}^{i}=\beta_{1}^{i} A_{i} E_{t x}(r)+\beta_{2}^{i} A_{i} E_{t x}(2 r)+\beta_{3}^{i} A_{i} E_{t x}(3 r) \\
\quad \text { for } j=1,2,3 \text { if } i-j<0 \text { then } \beta_{j}^{i}=0 \\
E_{R X}^{i}=\beta_{1}^{i+1} A_{i+1} E_{r x}(r)+\beta_{2}^{i+2} A_{i+2} E_{r x}(2 r)+\beta_{3}^{i+3} A_{i+3} E_{r x}(3 r) \\
\quad \text { for } j=1,2,3 i f i+j>K \text { then } \beta_{j}^{i+j}=0
\end{gathered}
$$

Thus, our optimization problem can be introduced as follows:

$$
\begin{aligned}
& \text { given } K, r, d_{t x-\max } \\
& \text { Find } \beta_{1}^{i}, \beta_{2}^{i}, \beta_{3}^{i} \forall i, 1 \leq i \leq K \\
& \text { such that } E^{1}=E^{2}=\ldots=E^{K} \\
& \text { subject to } \\
& \beta_{1}^{i}+\beta_{2}^{i}+\beta_{3}^{i}=1, \forall i, 1 \leq i \leq k
\end{aligned}
$$

To summarize, our main objective is to evenly distribute the data dissemination load among all underwater sensors. For this purpose, we deal with two different study cases. In the first case, we assume that each underwater sensor can dynamically adjust its transmission range such that the $2 r$ away neighbor is directly reached. In the second case study, we suppose that each sensor node can even directly reach the $3 r$ away neighbor. Note that, in the first case, two possible transmission ranges can be adopted by each sensor namely $r$ and $2 r$. However, in the second case, each node is able to select among three transmission ranges, $r, 2 r$ and $3 r$. Our objective, in both study cases, is to determine for each possible transmission range (namely possible next hop) the associated load weight that evenly distribute the energy expenditure among sensors. By summing at each sensor node the total received traffic with the generated one, we derive the optimal load weight for each potential next hop that balance energy depletion among sensor nodes.

In what follows, we denote $E_{r x}(j r)$ as $E_{r x}^{j}, E_{t x}(j r)$ as $E_{t x}^{j}$ and the vector $\beta^{i}$ refers to $\left(\beta_{l}^{i}\right)_{1 \leq l \leq 2}$ for the first case and to $\left(\beta_{l}^{i}\right)_{1 \leq l \leq 3}$ for the second case.

\section{B. Proposed Solution}

It is worth noting that, in both of the aforementioned study cases, the perfect uniform energy depletion is impossible to establish. Indeed, in the derived optimization of Eq. (10) and Eq. (15) the number of unknowns is much greater than the number of equations. In fact, for the first study case (where $d_{t x-\max } \in\{r, 2 r\}$ ), at each corona $i$ we have to determine 2 unknown variables $\left(\beta_{1}^{i}\right.$ and $\beta_{2}^{i}$ ) resulting in a total number of unknowns equal to $2 K$ but with only $K-1$ equations $\left(E^{1}=E^{2}=\ldots=E^{K}\right)$. In the same way, for the second study case, where $d_{t x-\max } \in\{r, 2 r, 3 r\}$, we have $(K-1)$ equations with a total number of unknowns equal to $3 K$. Consequently, both optimization problems are impossible to solve and so is the optimal energy balancing among coronas. For this reason, we strive for approaching the perfect uniform energy depletion. To do so, we slightly deviate our goal to the one of minimizing the maximum energy consumption among all coronas. Consequently both of our optimizations can be reformulated as follows:

$$
\begin{aligned}
& \text { given } K, r, d_{t x-\max } \\
& \text { Find } \beta^{i}, \forall i, 1 \leq i \leq K \\
& \min _{\beta^{i}} \max _{1 \leq i \leq K} E^{i} \\
& \text { subject to } \\
& \sum_{j=1}^{2} \beta_{j}^{i}=1 \\
& \beta^{i} \geq 0 \\
& \text { given } K, \quad r, d_{t x-\max } \\
& \text { Find } \beta^{i}, \forall i, 1 \leq i \leq K \\
& \min _{\beta^{i}} \max _{1 \leq i \leq K} E^{i} \\
& \text { subject to } \\
& \sum_{j=1}^{3} \beta_{j}^{i}=1 \\
& \beta^{i} \geq 0
\end{aligned}
$$

This constrained nonlinear optimization problem can be easily solved using 'fmincon' function in the Matlab optimization toolbox.

\section{Performance Evaluation}

In this section, we present an exhaustive comparison study between our balanced routing solution with $d_{t x-\max } \in$ 


\begin{tabular}{|l|l|}
\hline Packet length $P_{l}$ & 1024 bits \\
\hline Initial Energy & $100 \mathrm{~J}$ \\
\hline Data Rate A & 0.08 packet/s \\
\hline$P_{r x}^{0}$ & $0.75 \mathrm{~W}$ \\
\hline
\end{tabular}

TABLE I

PARAMETERS SETTING

$\{r, 2 r\}$ and $d_{t x-\max } \in\{r, 2 r, 3 r\}$ and the nominal communication range based data forwarding [2] with $d_{t x-\max }=r$. The results are derived numerically. We consider a circular sensor field of radius $R$, centered at the sink and partitioned into disjoint concentric coronas of constant fixed width $r$. Recall that in our network model, we consider a continuous monitoring application where each underwater sensor nodes periodically generates $A$ reports per unit of time to be forwarded to the sink over several hops. Each generated packet is forwarded from the source node to the target sink by crossing sensors located in the same wedge. At each hop, the local sensor node has to forward, not only its own generated traffic but also the route-through traffic. The parameters' settings in our analysis are given in Table. I.

We start by analyzing the performance of our balanced routing strategy for a circular sensor field of radius $R=1000 \mathrm{~m}$ and corona width $r=100 \mathrm{~m}$ resulting in a total number of coronas equal to 10 . Each sensor in each sector is generating of traffic rate of 0.08 packet/s. We study the outcome of using variable transmission range $\left(d_{t x-\max } \in\{r, 2 r\}\right.$ and $\left.d_{t x-\max } \in\{r, 2 r, 3 r\}\right)$ on both packet load distribution and energy consumption for every corona. First, let us discover $\beta=\left(\beta^{i}\right)_{1 \leq i \leq 10}$ matrix of our balanced routing scheme when $d_{t x-\max } \in\{r, 2 r\}$ and $d_{t x-\max } \in\{r, 2 r, 3 r\}$. We point out that the $\beta$ matrix is numerically obtained with the aim to evenly distribute the energy consumption among different coronas. Table II and table III report the $\beta^{i}$ vectors for each corona when $d_{t x-\max } \in\{r, 2 r\}$ and $d_{t x-\max } \in\{r, 2 r, 3 r\}$. Accordingly, in order to reduce the energy consumption gap between different coronas, most of the traffic should be sent to the furthest possible corona namely, the 2-hop away corona and 3-hop away corona respectively. In other words, to achieve a balanced energy consumption among different coronas, most of the accumulated traffic should be forwarded using $d_{t x-\max }=2 r=200 m$ and $d_{t x-\max }=3 r=300 m$ respectively. According to Table. II and Table. III the packet load distribution is shown in Fig. 2. It is worth noting that, adopting a nominal communication range based data forwarding with $d_{t x-\max }=r$ leads to a total traffic of 0.8 packet/s at sensors in corona 1 . This amount of accumulated traffic at corona 1 is highly decreased (around 0.3 packet/s) with our balanced routing solution when $d_{t x-\max } \in\{r, 2 r\}$, a further decrease is achieved when $d_{t x-\max } \in\{r, 2 r, 3 r\}$. This gain is more importantly highlighted in Fig. 3. In fact, Fig. 3 shows the energy consumption for each sensor in the corresponding corona. Accordingly, a $77 \%$ of energy saving is achieved at corona 1 when $d_{t x-\max } \in\{r, 2 r\}$ and a $87.5 \%$ of energy saving is obtained when $d_{t x-\max } \in\{r, 2 r, 3 r\}$ compared to the nominal based data forwarding. It is worth

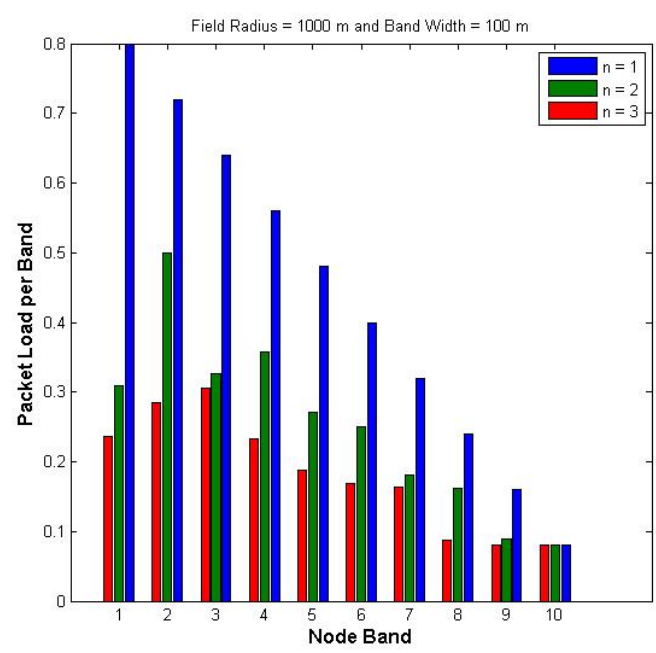

Fig. 2. Packet load distribution when $R=1000 \mathrm{~m}$ and $r=100 \mathrm{~m}$.

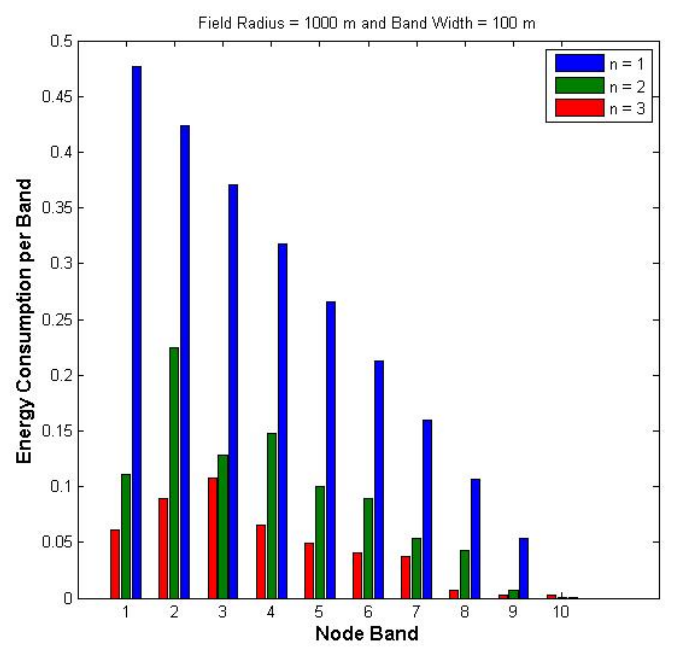

Fig. 3. Energy consumption per corona when $R=1000 \mathrm{~m}$ and $r=100 \mathrm{~m}$.

noting that using our balanced routing strategy leads to a maximum energy expenditure of $0.22 \mathrm{~W}$ at sensors in corona 2 and $0.11 W$ at sensor in corona 3 when $d_{t x-\max } \in\{r, 2 r\}$ and $d_{t x-\max } \in\{r, 2 r, 3 r\}$ respectively. Consequently, an energy saving of $54 \%$ and $77 \%$ are accomplished thanks to our balanced routing scheme when $d_{t x-\max } \in\{r, 2 r\}$ and $d_{t x-\max } \in\{r, 2 r, 3 r\}$ respectively.

We now turn to the task of evaluating the efficiency of our balanced routing solution $\left(d_{t x-\max } \in\{r, 2 r\}\right.$ and $\left.d_{t x-\max } \in\{r, 2 r, 3 r\}\right) \quad$ over the nominal communication range based data forwarding $\left(d_{t x-\max }=r\right)$ for different field radiuses as well as different corona widths. To do so, we aim at assessing the gain introduced by our balanced routing strategy in terms of energy consumption and network lifetime. It is worth pointing out that, the energy consumption comparison study considers the maximum consumed amount of energy among all coronas. To conduct a network lifetime comparison study, we first 
TABLE II

$\beta$ MATRIX WHEN $d_{t x-\max } \in\{r, 2 r\}$.

\begin{tabular}{|l|l|l|}
\hline Corona $i$ & Corona $(i-1)$ & Corona $(i-2)$ \\
\hline Corona 1 & 1 & 0 \\
\hline Corona 2 & 0.02 & 0.98 \\
\hline Corona 3 & 0.32 & 0.68 \\
\hline Corona 4 & 0.12 & 0.88 \\
\hline Corona 5 & 0.25 & 0.75 \\
\hline Corona 6 & 0.16 & 0.84 \\
\hline Corona 7 & 0.17 & 0.83 \\
\hline Corona 8 & 0.14 & 0.86 \\
\hline Corona 9 & 0.12 & 0.88 \\
\hline Corona 10 & 0.1 & 0.9 \\
\hline
\end{tabular}

TABLE III

$\beta$ MATRIX WHEN $d_{t x-\max } \in\{r, 2 r, 3 r\}$.

\begin{tabular}{|l|l|l|l|}
\hline Corona $i$ & Corona $(i-1)$ & Corona $(i-2)$ & Corona $(i-3)$ \\
\hline Corona 1 & 1 & 0 & 0 \\
\hline Corona 2 & 0.02 & 0.98 & 0 \\
\hline Corona 3 & 0.03 & 0.05 & 0.92 \\
\hline Corona 4 & 0.12 & 0.28 & 0.6 \\
\hline Corona 5 & 0 & 0.31 & 0.69 \\
\hline Corona 6 & 0 & 0.17 & 0.83 \\
\hline Corona 7 & 0 & 0.24 & 0.76 \\
\hline Corona 8 & 0 & 0.23 & 0.77 \\
\hline Corona 9 & 0 & 0.14 & 0.86 \\
\hline Corona 10 & 0 & 0.1 & 0.9 \\
\hline
\end{tabular}

start by defining the network lifespan as the time for the first underwater sensor in the network to deplete its battery power. More precisely, the network lifetime is derived as follows

$$
T_{\text {net_lifetime }}=\frac{E_{\text {init }}}{\max _{U \in \text { corona_nodes }} E(U)}
$$

where $E_{\text {init }}$ is the initially provided amount of energy to each underwater sensor and $U$ refers to an arbitrary sensor node in the network field under study.

Fig. 4 depicts the energy consumption for different field radius values when the corona width remains fixed and equal to $r=100 \mathrm{~m}$. As expected, the energy consumption increases with the field radius since the total number of coronas rises. Therefore and as shown in Fig. 4, as the field radius increases, the network lifetime is reduced. More importantly, our balanced routing solution when $d_{t x-\max } \in\{r, 2 r\}$ considerably reduces the energy expenditure compared to the nominal communication range based data forwarding. Indeed, when $d_{t x-\max }$ varies in $\{r, 2 r\}$, up to $64 \%$ of energy saving is achieved for a field radius of $500 \mathrm{~m}$ and a minimum energy saving of $52 \%$ is guaranteed for each possible field radius. Moreover, a further energy saving is achieved when $d_{t x-\max }$ varies in $\{r, 2 r, 3 r\}$. As such, can we conclude that as we extend the transmission range, the energy saving is increased? To answer this question, we evaluate the energy consumption for various corona widths.

To achieve this, we consider a fixed field radius of $3000 \mathrm{~m}$ while varying the corona width from $100 \mathrm{~m}$ to $900 \mathrm{~m}$. According to Figs. 7 and 8, for each configuration (i.e., $d_{t x-\max }=r$, $d_{t x-\max } \in\{r, 2 r\}$ and $\left.d_{t x-\max } \in\{r, 2 r, 3 r\}\right)$ there is an optimal value of the corona width $C w_{\text {opt }}$ which provides the minimum energy consumption and hence the maximum network lifetime. Indeed, when the corona width increases up to $C w_{\text {opt }}$ the energy expenditure decreases. In fact, rising the

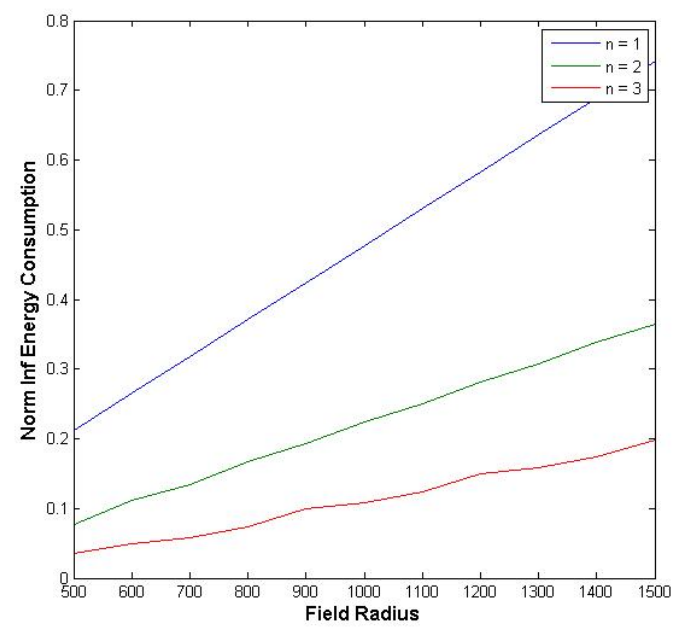

Fig. 4. Energy consumption for different field radius when $r=100 \mathrm{~m}$.

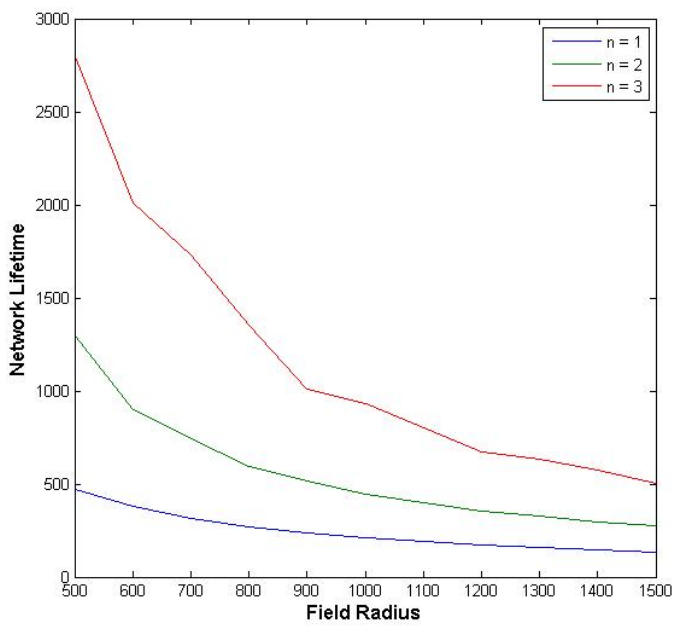

Fig. 5. Network lifetime for different field radius when $r=100 \mathrm{~m}$.

corona width reduces the number of coronas and consequently the packet load is reduced. However when the corona width exceeds $C w_{\text {opt }}$ the energy expenditure increases even though the packet load is reduced. The reason behind this is the non linearity of transmission power as function of distance. Indeed, according to Fig. 6, for high value of transmission range, the transmission power is exponentially increasing leading consequently to high energy depletion.

Comparing our balanced routing schemes with the nominal range forwarding for different values of corona width, we observe that the three schemes behave differently. Indeed, for a corona width equals $200 \mathrm{~m}$, the minimum energy consumption is achieved by our balanced routing strategy with $d_{t x-\max } \in$ $\{r, 2 r, 3 r\}$ while the maximum energy depletion is achieved by the nominal communication range based data forwarding. However, for a corona width equals $700 \mathrm{~m}$, the least energy consumption is achieved with the nominal communication range based data forwarding while the highest energy consumption is fulfilled with our balanced routing strategy when 


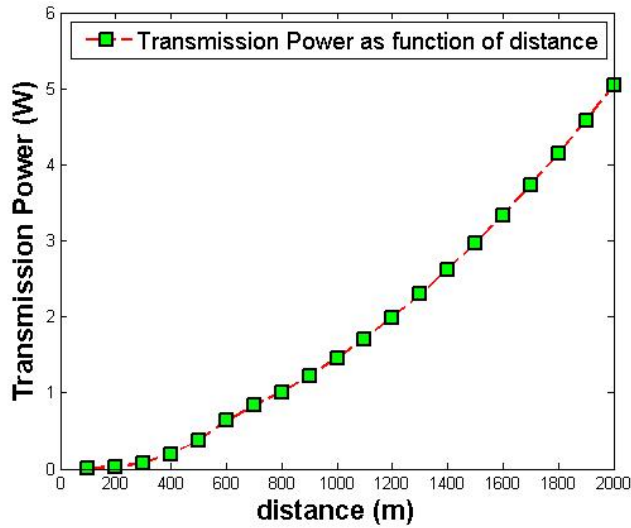

Fig. 6. Transmission power for various transmission distance.

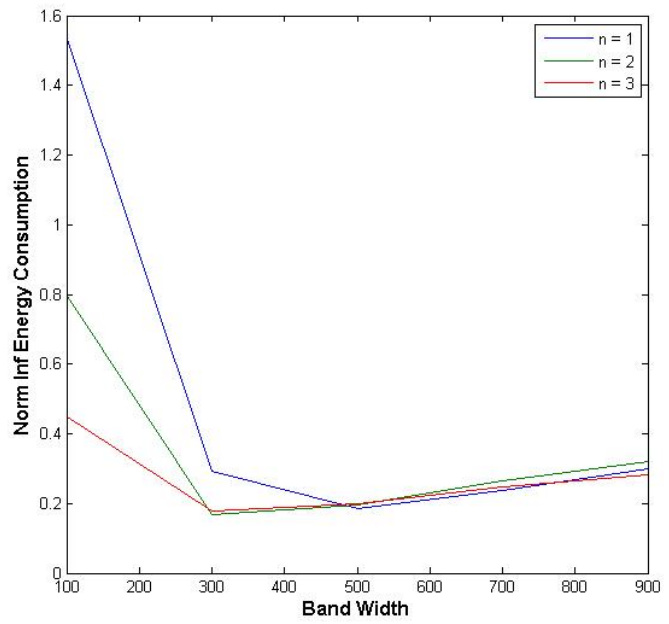

Fig. 7. Energy consumption for various corona width when $R=3000 \mathrm{~m}$.

$d_{t x-\max }$ varies within $\{r, 2 r\}$. As a conclusion, we can state that for each corona width there is an optimal configuration (namely; $d_{t x-\max }=r, d_{t x-\max } \in\{r, 2 r\}$ and $d_{t x-\max } \in$ $\{r, 2 r, 3 r\}$ ) for which the energy consumption is minimized. Moreover, for each configuration, there is an optimal corona width for which the network lifetime is optimized. Combining both results, and according to Figs. 7 and 8 , the maximum network lifetime is achieved by our balanced routing solution when $d_{t x-\max } \in\{r, 2 r\}$ for a corona width equal to $300 \mathrm{~m}$.

\section{CONCLUSION}

UW-ASNs necessitates protocols that smoothly and uniformly consume the limited sensor's battery budget among all the sensor in order to maximize the network lifespan. For this purpose, we proposed an underwater sensor network deployment pattern along with the associated routing scheme that aim at evenly consume the energy capacity among all sensors in the network. Indeed, our routing strategy supposes that every sensor node is endowed with multiple transmission ranges that can be appropriately adjusted to sent or forward the periodically generated data. More precisely, we determined

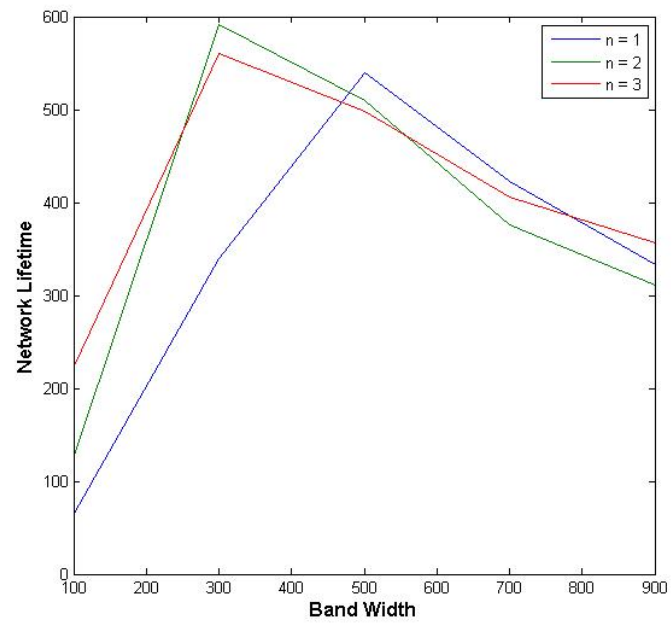

Fig. 8. Network lifetime for various corona width when $R=3000 \mathrm{~m}$.

for each source sensor the set of possible next hops with the associated transmission range and associated load weight that approach the optimal uniform energy consumption among all underwater sensors. For this purpose, we analytically state an optimization problem that has been numerically solved to derive for each source sensor the appropriate load weight along with the associated transmission range. Numerical results show that significant energy conservation is achieved by our routing scheme compared to the nominal communication range based data forwarding.

\section{REFERENCES}

[1] I. F. Akyildiz, D. Pompili, and T. Melodia, Underwater Acoustic Sensor Networks: Research Challenges, Ad Hoc Networks (Elsevier), vol. 3, no. 3, pp. 257-279, May 2005.

[2] H. Ammari and S.K. Das, Promoting Heterogeneity, Mobility, and Energy-Aware Voronoi Diagram in Wireless Sensor Networks, IEEE Transactions on Parallel and Distributed Systems, vol. 19, pp. 995-008, 2008.

[3] J. Li and P. Mohapatra, Analytical modeling and mitigation techniques for the energy hole problem in sensor networks, Pervasive and Mobile Computing (Elsevier), vol. 3, pp. 233-254, 2007.

[4] H. Ammari, Investigating the Energy Sink-Hole Problem in Connected k-Covered Wireless Sensor Networks, IEEE Transactions on Computers, issue 99, pp. 1-14, 2013.

[5] F. Jiang, D. Huang, C. Yang and K. Wang, Mitigation techniques for the energy hole problem in sensor networks using N-policy M/G/1 queuing models,International Conference on Theory, Technologies and Applications, Frontier Computing, 2010.

[6] X. Wu, G. Chen, and S. K. Das, Avoiding energy holes in wireless sensor networks with nonuniform node distribution, IEEE Transactions on Parallel and Distributed Systems, vol. 19, MAY 2008.

[7] M. Stojanovic, "On the relationship between capacity and distance in an underwater acoustic communication channel," ACM SIGMOBILE Mobile Comput. Commun. Rev., vol. 11, no. 4, pp. 34-43, Oct. 2007. 